\title{
Genetically modified Mosquitoes-blessing or curse
}

\section{Burden of disease on population}

Malaria has been a problem in India for centuries. Details of this disease can be found even in the ancient Indian medical literature like the Atharva Veda and Charaka Samhita. Globally, malaria kills up to a million people each year, mostly children. Between 300 million and 500 million cases of malaria occur annually and an estimated 40 per cent of the world's population live in malaria areas. ${ }^{1}$ According to the World Malaria Report 2014, 22\% (275.5m) of India's population live in high transmission ( $>1$ case per 1000 population) areas, $67 \%$ $(838.9 \mathrm{~m})$ live in low transmission $(0-1$ cases per 1000 population) areas and $11 \%(137.7 \mathrm{~m})$ live in malaria-free ( 0 cases) areas. ${ }^{2}$ In 2013 , 0.88 million cases have been recorded, with 128 million tests being conducted on the suspected cases, with $P$. falciparum causing 53\% and $P$. vivax causing $47 \%$ of the infections. The incidence of malaria in India accounted for $58 \%$ of cases in the South East Asia Region of WHO. ${ }^{3}$ At present, official figures for malaria in India, available at NVBDCP, indicate 0.7-1.6 million confirmed cases and 400-1,000 deaths annually. ${ }^{4}$

\section{History of malarial parasite}

In 1870 the discoveries of Pasteur and Koch had precipitated a search for a bacterial cause for many diseases including malaria. In 1880 , Laveran meticulously examined the blood of 200patients and in 148 observed the crescentic bodies in all cases of malaria but never in those without malaria. He also noted that quinine removed these stages from the blood. Laveran quickly realized that he had found a parasitic protozoan which he called Oscillariamalariae..$^{5}$ In initial $19^{\text {th }}$ century, it was known that malaria was caused by a protozoan parasite that invaded and multiplied in red blood cells and, after a lot of confusion, that there were three species with specific periodicities and other characteristics responsible for benign tertian (Haemamoebavivax), malignant tertian (Laveraniamalariae) and quartan (Haemamoebamalariae) malaria now respectively Plasmodium vivax, $P$. falciparum and $P$. malariae. ${ }^{6}$

\section{Genetically modified mosquitoes}

A malaria-free mosquito has been created by scientists using a genetic technology that causes the disease-free trait to be inherited by virtually all its offspring - raising the possibility of eradicating malaria within a single breeding season. The genetically-engineered mosquito is incapable of transmitting malaria to humans and can pass on its disease immunity to 99.5 per cent of its progeny, according to a study. Previous experiments on fruit flies have shown that gene drives can caused the rapid spread of genes within a breeding population of captive flying insects. Now scientists have demonstrated that gene drives combined with a powerful gene-editing technique called Crispr/Cas9 can cause the rapid spread of genes for malaria resistance in a laboratory population of the Anopheles stephensi mosquito, one of the principal carriers of malaria in Asia. Together, they created a "cassette" of genes that including an antimalarial antibody gene and the Cas9 enzyme for cutting and splicing DNA so that the antibody gene was transmitted from one chromosome to another in the same mosquito embryo. ${ }^{7}$ The genetically modified mosquito carries extra genes for antibodies that block the development of the malaria parasite within the insect and so prevent the disease from being transmitted to people when mosquitoes feed on human blood. The level of mosquito
Volume 5 Issue I - 2018

\author{
Nidhi Sharma, Sanjeev Kumar Jain \\ Teerthanker Mahaveer Medical College and Research Centre, \\ India
}

Correspondence: Sanjeev Kumar Jain, Vice Dean and Professor of Anatomy Teerthanker Mahaveer Medical College and Research Centre, Moradabad, UP 24400I India, Email drskjain2005@rediffmail.com

Received: April 17, 2017| Published: August 15, 2018

suppression that's needed to stop disease transmission depends on a wide variety of factors, including the mosquito population, the human population, the amount of disease already circulating, and the proportion of people previously infected, and therefore immune. ${ }^{8}$

\section{Modification at genetic level}

The A. gambiae Rel2S was PCR-amplified and separately ligated to the A. gambiae carboxypeptidase $\mathrm{A}(\mathrm{AgCp})$ and vitellogenin $1(\mathrm{AgVg})$ promoters in independent constructs, and the terminator sequence of the A. gambiae trypsin gene (TryT) was ligated downstream of Rel2. These two cassettes, AgCp-Rel2-TryT and AgCp-Rel2-TryT, were separately cloned into the piggy Bac-based plasmids pBac [3xP3EGFPafm] and $\mathrm{pBac}$ [3xP3-DsRedafm] containing the eye-specific $3 \times 3$ promoter-driven GFP or DsRed as selection markers for the screening of transgenic mosquitoes. ${ }^{9}$

\section{Transformation of vector}

For the mosquito germ-line transformation, they used the $\mathrm{pBac}$ [3xP3-EGFPafm] and $\mathrm{pBac}$ [3xP3-DsRedafm] transformation vectors containing an eye-specific promoter $(3 \times \mathrm{P} 3)$ in front of the TATA box. The active form (1840 bp) of the A. gambiae Rel2 (Rel2S) lacking the ankyrin repeats and death domains was first cloned into the pBluescript vector at the EcoRI site (Stratagene). The Rel2 primers used for amplifying the PCR product from A. gambiae cDNA were: 5'-GCAGTGGTCAGTGTTGGAGAG-3' (forward primer) and $5^{\prime}$-TTCCGAGTTACAGGGGAA GTC-3' (reverse primer). putative terminator region of trypsin was obtained by PCR from the vector $\mathrm{pENTR}$-carboxypeptidase P-antryp $1 \mathrm{~T} .{ }^{10}$

\section{Success stories}

There is success story in agriculture-sterility by genetic modification helped wipe out the New World screwworm in the 1960s, which once devastated livestock in the United States. Similar methods have been used to successfully fight fruit flies in the United States. Sterility as the end-result of genetic modification has other benefits. For one, it basically makes a species breed itself out of existencerather than introducing a new kind of pest that continues to pass along its altered genes for generations. "There's an inherent safety aspect to what we're doing," McKemey said. "The very thing we're releasing is designed to disappear from the environment without a trace."11 


\section{Unknown fear}

You need to be concerned about what you don't know," said Ravi Durvasula, a professor of Medicine and Infectious Diseases at University of New Mexico School of Medicine. The researchers who are against this technology states that what if the desired gene mutation doesn't take, and people end up releasing mass quantities of new mosquitoes that end up making the other viral infections like HIV, Zikaetc problem worse? It's also unlikely that this mutant is going to somehow mutate again and give you something undesirable. What if, for example, the gene modification ends up altering a mosquito's behavior - making it more aggressive, or changing its host preference? You can start to fantasize about every possible fate of that gene, but it's impossible to test all of that in a lab," he said. "And once you've released a trait into a population, there cannot be a recall. This is what scares researchers. They get creeped out by these things. ${ }^{8}$

\section{Acknowledgements}

None.

\section{Conflict of interest}

The authors declare that there is no conflict of interest.

\section{References}

1. Richard Tren. Malaria and Climate Change. Working Papers Series: Julian Simon Centre for Policy Research. 2002.
2. Kumar A, Valecha $\mathrm{N}$, Jain $\mathrm{T}$, et al. Burden of Malaria in India: Retrospective and Prospective View. Am J Trop Med Hyg. 2007;77(6 Suppl):69-78

3. WHO. World Malaria Report. Geneva: WHO; 2014.

4. Dhingra N, Jha P, Sharma VP, et al. Adult and child malaria mortality in India: a nationally representative mortality survey. Lancet. 2010;376(9754):1768-1774.

5. Stephens JWW. A new malaria parasite of man. Ann Trop Med Parasitol. 2011;16(40:383-388.

6. MacCallum WG. On the flagellated form of the malarial parasite. Lancet. $1897 ; 1240-1241$

7. Huang $\mathrm{Y}$, Magori K, Lloyd AL, et al. Introducing desirable transgenes into insect populations using Y-linked meiotic drive - a theoretical assessment. Evolution. 2007;61(4):717-726.

8. Caraballo H, King K. Emergency department management of mosquitoborne illness: malaria, dengue, and West Nile virus. Emerg Med Pract. 2014;16(5):1-23.

9. Sinha S, Medhi B, Sehgal R. Challenges of drug-resistant malaria. Parasite. 2014;21:61.

10. Fraiture M, Baxter RH, Steinert S, et al. Two mosquito LRR proteins function as complement control factors in the TEP1-mediated killing of Plasmodium. Cell Host Microbe. 2009;5(3):273-284.

11. Shahabuddin M. Plasmodium ookinete development in the mosquito midgut: a case of reciprocal manipulation. Parasitology. 1998;116(Suppl):S83-S93. 